\title{
Reoperation following the use of non-standardized procedures for malignant parotid tumors
}

\author{
XIANGMIN ZHANG $^{1 *}$, XIANGFU ZENG ${ }^{2 *}$, XIAOLIN LAN ${ }^{1 *}$, JING HUANG $^{3}$, KEQING LUO $^{1}$, \\ KEQIANG TIAN ${ }^{1}$, XIUHONG WU $^{4}$, FUFU XIAO $^{1}$ and SHAOJIN LI $^{3}$ \\ ${ }^{1}$ Department of Head and Neck, Tumor Hospital of Ganzhou; ${ }^{2}$ Department of General Surgery, First Affiliated Hospital \\ of Gannan Medical University; ${ }^{3}$ Ganzhou Institute of Cancer Research, ${ }^{4}$ Department of Radiation Oncology, \\ Tumor Hospital of Ganzhou, Ganzhou, Jiangxi 341000, P.R. China
}

Received February 28, 2016; Accepted July 27, 2017

DOI: $10.3892 / 01.2017 .7020$

\begin{abstract}
Non-standardized or conservative procedures are employed when parotid tumors involving the facial nerve or parotid carcinoma are misdiagnosed as benign parotid tumors prior to or during surgery. Remedial measures are usually required when the pathological diagnosis of a malignant parotid tumor is confirmed following surgery. The aim of the present study was to systematically evaluate reoperation subsequent to treatment with non-standardized procedures for malignant parotid tumors, and to explore the preoperative diagnoses, the primary procedure selection and the necessity of reoperation following non-standardized procedures in malignant parotid tumors. A total of 30 patients who met the inclusion criteria, were diagnosed with a malignant parotid tumor and underwent reoperation following the use of a non-standardized procedure were included in the present study. Surgical conditions and clinical data were analyzed. Among the patients with a malignant parotid tumor who underwent reoperation subsequent to a non-standardized procedure, the incidence of residual tumor, as confirmed by pathological examination, was $63.3 \%$ (19/30). The intact facial nerve preservation rate was $83.3 \%$ $(25 / 30)$, the facial nerve branch resection rate was $6.7 \%(2 / 30)$, the facial partial nerve resection rate was $6.7 \%(2 / 30)$ and the facial nerve resection rate was $3.3 \%$ (1/30). In total, 3 patients underwent facial nerve reconstruction, 3 patients underwent a local flap repair of skin defects in the parotid region and 3 patients underwent pectoralis major muscle flap repair. The current findings indicate that the qualitative diagnosis of malignant parotid tumors prior to surgery is difficult, there is a high incidence of residual tumor following non-standardized
\end{abstract}

Correspondence to: Professor Shaojin Li, Ganzhou Institute of Cancer Research, 19 HuaYuan Qian Road, Ganzhou, Jiangxi 341000, P.R. China

E-mail: 1sj1362@126.com

*Contributed equally

Key words: parotid tumor, malignancy, reoperation procedures, and that reoperation in a timely manner is required in such cases.

\section{Introduction}

Salivary gland cancer is rare, with an overall incidence of between 2.5 and 3.0 cases per 100,000 individuals globally, accounting for $<3.0-5.0 \%$ of all head and neck cancer (1). Almost $80 \%$ of salivary gland tumor occurs in the parotid gland, which are challenging to differentially diagnose (2). Mucoepidermoid carcinoma is the most common type of parotid gland cancer, which is associated with favorable prognosis, with a 5-year overall survival of $79 \%$ depending on clinical stage and grade (3). Other pathological types of salivary gland cancer include adenoid cystic carcinoma, acinic cell carcinoma and salivary ductal carcinoma (4). Surgery coupled with potential use of adjuvant radiation therapy and chemotherapy are performed for the treatment of salivary gland malignancies (5). The clinical manifestations of parotid tumors are variable and their pathological features are complex. The selection of the optimal surgical procedure presents its own challenges due to difficulties differentiating between malignant and benign tumors through general clinical examination (6). A lack of pathological examination prior to or during surgery, or misdiagnosis by preoperative punch biopsy or intraoperative frozen section, may lead to the selection of an inappropriate surgical procedure $(7,8)$. Reoperation, with caution, is required in cases where a diagnosis of malignant parotid tumor is confirmed subsequent to surgery by pathological examination (9).

The present study reviewed the cases of 30 patients with malignant parotid tumor who underwent reoperation subsequent to the use of a non-standardized procedure. The study aimed to systematically evaluate the use of reoperation, explore the preoperative diagnosis, selection of primary procedure and necessity of reoperation subsequent to a non-standardized primary procedure, and provide a reference for the clinical diagnosis and treatment of patients with malignant parotid tumors.

\section{Patients and methods}

Ethical statement. The Ethics Committee of the Tumor Hospital of Ganzhou Review Board (Ganzhou, China) approved the 
present study protocol (no. 20080203). The present study was conducted in accordance with the Declaration of Helsinki with regard to research involving human subjects, and all patients provided written informed consent to participate following explanation of the nature of the study.

Inclusion criteria. The inclusion criteria were as follows: i) The patient had a malignant parotid tumor and was recommended by their primary surgeon for transfer to a higher tier hospital for reoperation; ii) the patient underwent a primary surgery comprising partial tumor resection, tumor enucleation and partial superficial lobe parotidectomy; iii) the patient underwent physical examination and enhanced computed tomography (CT) scans that revealed residual tumor, or prompted suspicion of residual tumor, at the primary tumor site or showed cervical lymph node enlargement; iv) the patient was aged between 18 and 70 years; v) the patient had a Karnofsky performance score of $>80$ (10); vi) the expected survival time of the patient was $>1$ year; and vii) the patient voluntarily signed informed consent forms.

Exclusion criteria. The exclusion criteria were as follows: i) $>3$ months had elapsed since the primary surgery; ii) the patient had any other malignant tumors of the head and neck; iii) the patient had a history of head and neck radiotherapy; and iv) the patient had severe heart, lung, liver or kidney disease.

Clinical data. Between January 2008 and December 2014, 30 consecutive patients [17 male and 13 female; median age, 47 (range, 18-69) years] with malignant parotid tumors, who underwent reoperation at the Tumor Hospital of Ganzhou and who met the inclusion criteria, were included in the present study. Patients had undergone either 1 or 2 prior procedures at other hospitals, with the time elapsed between the primary surgery and the reoperation ranging from 7 to 83 days (Table I).

Reoperation procedures. All procedures were performed by two experienced, qualified surgeons. Total parotidectomy was performed on all patients for management of the primary tumor. With regard to lymph node management, prophylactic neck dissection was not required for patients with low-grade malignant parotid tumors of preoperative cNO stage if no metastasis-positive level II lymph nodes were observed intraoperatively; while selective neck dissection of levels I-III or I-IV was required if lymph nodes were revealed to be positive for metastasis by intraoperative pathological examination of frozen sections $(11,12)$ : Freezing for $2 \mathrm{~min}$ at between -15 and $-20^{\circ} \mathrm{C}$ and prepared to a section thickness of 4.0-5.0 $\mu \mathrm{m}$ on a freezing microtome (Leica CM1950; Leica Microsystems $\mathrm{GmbH}$, Wetzlar, Germany). Tissue was fixed with AF fixation fluid (75 ml 95\% ethanol with $10 \mathrm{ml} \mathrm{40 \%} \mathrm{formaldehyde)}$ for $30 \mathrm{sec}$ at room temperature. Sections were stained with hematoxylin-eosin for $15 \mathrm{~min}$ at $60^{\circ} \mathrm{C}$ and evaluated under magnification, x40 (Olympus BX-53; Olympus Corporation, Tokyo, Japan). Selective or functional neck dissection was performed in patients with undifferentiated carcinoma, poorly differentiated mucoepidermoid carcinoma, squamous cell carcinoma, adenocarcinoma or cystadenocarcinoma at $\mathrm{cN} 0$ stage. For patients identified to have cervical lymph node enlargement preoperatively by physical examination or enhanced CT scan, a functional or radical neck dissection of the level I-IV nodes was performed according to lymph node status, as described above. For patients with tumor invasion of the skin in the parotid region, flap repair surgery was performed according to the individual skin defect conditions (13-15). All of the reoperation specimens were confirmed by conventional pathological paraffin sections and immunohistochemistry examination.

Facial nerve procedures. En bloc resection of the tumor with the facial nerve was performed if facial paralysis was present prior to surgery. If the tumor was attached to the nerve, the nerve was preserved if separation was possible, postoperative adjuvant radiotherapy (60 Gy in 30 fractions for 6 weeks) was administered. The facial nerve was excised in the event that separation from the tumor mass was difficult, or if the facial nerve was confirmed to pass through the tumor (16-18). Following partial or complete excision of the facial nerve, facial nerve reconstruction was performed if possible $(19,20)$. The House-Brackmann facial nerve grading system was used to assess facial nerve function following its reconstruction (21).

Radiation therapy. All patients who underwent reoperation required supplementary postoperative radiation therapy (22). Conventional radiotherapy or intensity-modulated radiation therapy were chosen according to the patient's own economic conditions following reoperation. Conventional radiation therapy was administered as follows (23-25): The anterior boundary of the parotid region was defined as the anterior edge of the masseter muscle, the posterior boundary was defined as the posterior edge of the mastoid process, the superior boundary was defined as the upper edge of the zygomatic arch and the inferior boundary was defined at the level $1.0 \mathrm{~cm}$ beneath the mandible. Neck lymphatic drainage area radiation was complementarily performed in patients confirmed with poorly differentiated tumors, late-stage tumors or neck lymph node metastases as previously described (26). The dosage of conventional external-beam radiation therapy was $60 \mathrm{~Gy}$ in 30 fractions for 6 weeks following reoperation; the maximal local dose was increased to 66-70 Gy in patients with positive surgical margins, and a dose of $\geq 66$ Gy was administered to patients with adenoid cystic carcinoma.

Intensity-modulated radiation therapy was administered as follows $(27,28)$ : The primary tumor site was defined as the gross tumor volume (GTV) of the tumor bed (GTVtb); the residual tumor was defined as the GTV; the subclinical stage and high-risk lymphatic drainage region was defined as clinical target volume (CTV) 1; and the prophylactic irradiation region of lymphatic drainage was defined as CTV2. The doses of the intensity-modulated radiation therapy following reoperation were as follows: 66-70 GY in 30-33 fractions (6-6.5 weeks) for GTV, 60-66 GY in 30-33 fractions (6-6.5 weeks) for GTVtb, 56-60 GY in 30-33 fractions (6-6.5 weeks) for CTV1, and $54 \mathrm{GY}$ in 30-33 fractions (6-6.5 weeks) for CTV2.

Observation indexes. The surgical wound healing process and facial nerve function recovery were monitored following surgery according to outpatient investigations, and any 
Table I. General clinical data of the patients $(n=30)$.

\begin{tabular}{|c|c|}
\hline Parameter & $\begin{array}{c}\text { Number of } \\
\text { patients, n }(\%)\end{array}$ \\
\hline \multicolumn{2}{|l|}{ Age, years } \\
\hline $18-39$ & $9(30.0)$ \\
\hline $40-49$ & $7(23.3)$ \\
\hline $50-59$ & $8(26.7)$ \\
\hline $60-70$ & $6(20.0)$ \\
\hline \multicolumn{2}{|l|}{ Sex } \\
\hline Male & $17(56.7)$ \\
\hline Female & $13(43.3)$ \\
\hline \multicolumn{2}{|l|}{ Karnofsky performance score } \\
\hline $80-89$ & $9(30.0)$ \\
\hline$\geq 90$ & $21(70.0)$ \\
\hline \multicolumn{2}{|l|}{ Pathological type } \\
\hline Mucoepidermoid carcinoma & $12(40.0)$ \\
\hline Adenoid cystic carcinoma & $7(23.3)$ \\
\hline Acinic cell carcinoma & $4(13.3)$ \\
\hline $\begin{array}{l}\text { Nonspecific poorly differentiated } \\
\text { adenocarcinoma }\end{array}$ & $1(3.3)$ \\
\hline Salivary duct carcinoma & $1(3.3)$ \\
\hline Malignancy of pleomorphic adenoma & $1(3.3)$ \\
\hline Lymphoepithelial carcinoma & $1(3.3)$ \\
\hline Epithelial-myoepithelial carcinoma & $1(3.3)$ \\
\hline Carcinoma ex pleomorphic adenoma & $1(3.3)$ \\
\hline Basal cell carcinoma & $1(3.3)$ \\
\hline \multicolumn{2}{|l|}{ Previous procedure } \\
\hline Partial tumor resection & $5(16.7)$ \\
\hline Tumor enucleation & $11(36.7)$ \\
\hline Partial superficial lobe parotidectomy & $13(43.3)$ \\
\hline Selective deep lobe parotidectomy & $1(3.3)$ \\
\hline \multicolumn{2}{|l|}{ Previous surgical complications } \\
\hline Facial nerve branch injury & $2(6.7)$ \\
\hline Parotid gland leakage & $2(6.7)$ \\
\hline \multicolumn{2}{|l|}{$\begin{array}{l}\text { Number of procedures performed } \\
\text { in other hospitals }\end{array}$} \\
\hline 1 & $26(86.7)$ \\
\hline 2 & $4(13.3)$ \\
\hline
\end{tabular}

complications were noted. Patients were also monitored for recurrences at the primary tumor site and neck lymph nodes. Patients were followed up at an interval of every 3 months for the first 2 years following treatment, every 6 months between years 2 and 5, and every 12 months thereafter. Follow-ups were performed from the day immediately following the completion of radiotherapy until December 31, 2015. The total follow-up durations ranged between 12 and 87 months.

Statistical analysis. All data analyses were performed using SPSS (version 22.0; IBM Corp., Armonk, NY, USA). The Kaplan-Meier estimator method was used for survival analysis.
Table II. Procedures undergone and complications experienced by the patients as part of reoperation $(n=30)$.

\begin{tabular}{lr}
\hline Procedure/complication type & $\begin{array}{c}\text { Number of } \\
\text { patients, } \mathrm{n}(\%)\end{array}$ \\
\hline $\begin{array}{l}\text { Primary tumor } \\
\text { Total parotidectomy }\end{array}$ & $30(100.0)$ \\
Cervical lymph nodes & \\
Selective neck dissection & $10(33.3)$ \\
Functional neck dissection & $6(20.0)$ \\
Radical neck dissection & $4(13.3)$ \\
Repair of skin defects & \\
Adjacent flap for transposition repair & $3(10.0)$ \\
Pectoralis major muscle flap for & $3(10.0)$ \\
transposition repair & \\
Facial nerve & \\
Resection of branch of the facial nerve & $2(6.7)$ \\
Partial resection of the facial nerve & $2(6.7)$ \\
Facial nerve resection & $1(3.3)$ \\
Repair and reconstruction of \\
great auricular nerve
\end{tabular}

\section{Results}

Patient treatments. A total of 30 patients underwent reoperation, and the incidence of residual tumor was $63.3 \%$ (19/30), as confirmed by conventional pathological paraffin sections and immunohistochemistry examination. The intact facial nerve preservation rate was $83.3 \%$ (25/30); 1 patient with complete facial nerve excision did not undergo a facial nerve graft procedure as complete tumor excision was considered to be the immediate priority, 1 patient with buccal branch excision did not undergo repair surgery for it did not affect important facial function, and the remaining 3 patients with facial nerve branch excision or partial excision underwent facial nerve reconstruction utilizing the great auricular nerve. Facial nerve function was recovered to House-Brackmann (27) grade II in 1 patient and grade III in 2 patients after a 3-month follow-up. A single patient experienced surgical field hemorrhage following surgery and underwent secondary debridement; 1 patient experienced local flap necrosis and an opened incision, and underwent secondary surgery with pectoralis major muscle flap transposition repair; and 2 patients experienced temporary facial paralysis and recovered within 3 months through nutritional support and acupuncture (Table II).

Follow-up. All 30 patients were followed-up. The follow-up rate was $100 \%$, with durations varying from 12 to 87 months. A total of 3 patients experienced local recurrence and 5 mortalities were reported, of which 2 patients with adenoid 


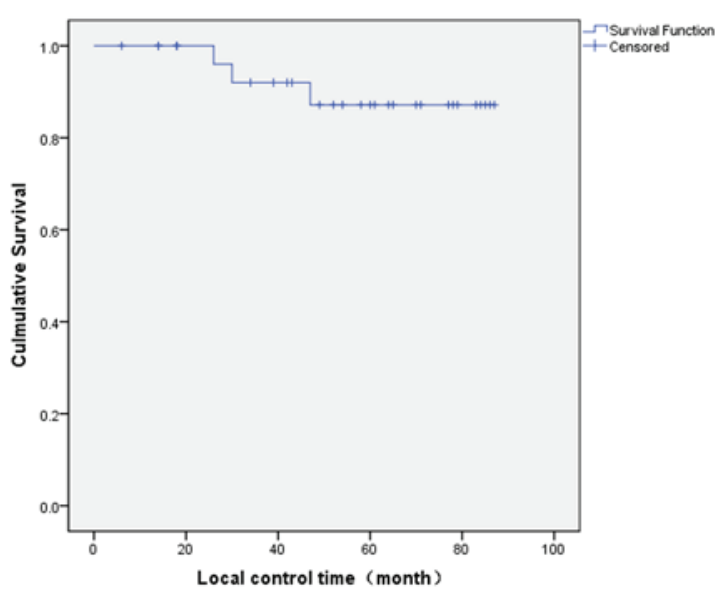

Figure 1. Kaplan-Meier estimator curve of local control rate. The 5-year local control rate was $87.2 \%$.

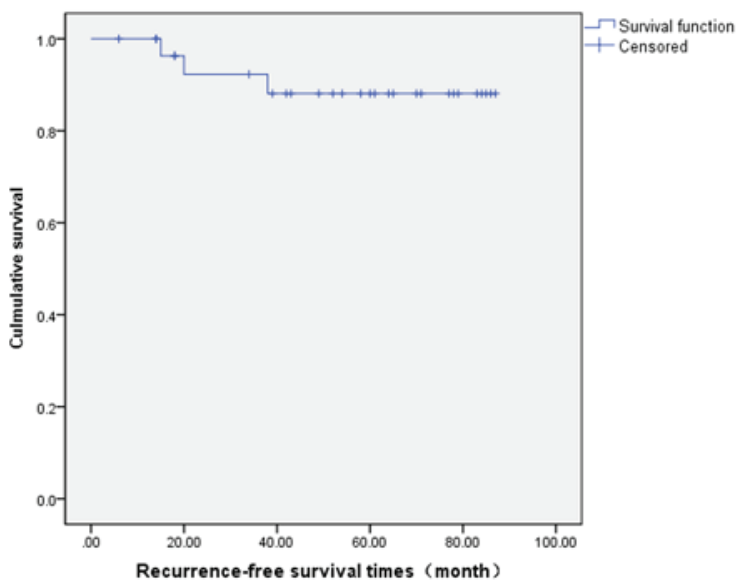

Figure 2. Kaplan-Meier estimator curve of recurrence-free survival. The 5 -year recurrence-free survival rate was $88.1 \%$.

cystic carcinoma succumbed to lung metastasis, 1 patient with atypical poorly differentiated adenocarcinoma succumbed to brain metastases, and 2 patients with poorly differentiated mucoepidermoid carcinoma succumbed to local recurrence. As analyzed using Kaplan-Meier estimator curves, the 5-year local control rate was $87.2 \%$ (Fig. 1), the 5-year recurrence-free survival rate was $88.1 \%$ (Fig. 2) and the 5-year overall survival rate was $81.7 \%$ (Fig. 3).

Typical case report. A 61-year-old male noticed a facial tumor below his right ear in March 2000. The tumor size was $2.5 \times 2.0 \mathrm{~cm}$. No other symptoms of discomfort were reported. Enlargement of the tumor under his right ear was noted, along with pruritus, in June 2010. Partial tumor excision was performed in Longkou Town Health Center (Ganzhou, China); however, the procedure details and postoperative pathological diagnosis were unknown. The residual tumor under the patient's right ear was determined to be progressively growing in May 2011. A reoperation was performed in the same health center in January 2013, partially excising the tumor. Following surgery, the surgical

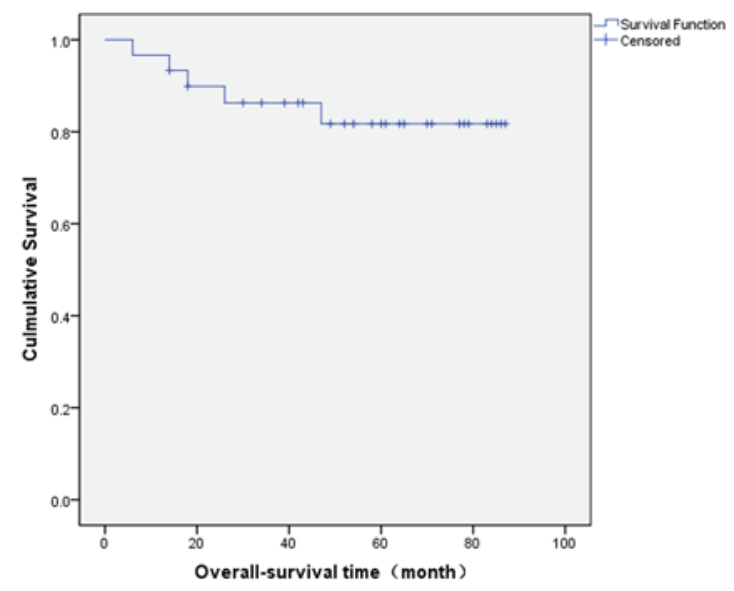

Figure 3. Kaplan-Meier estimator curve of overall survival. The 5-year overall survival rate was $81.7 \%$.

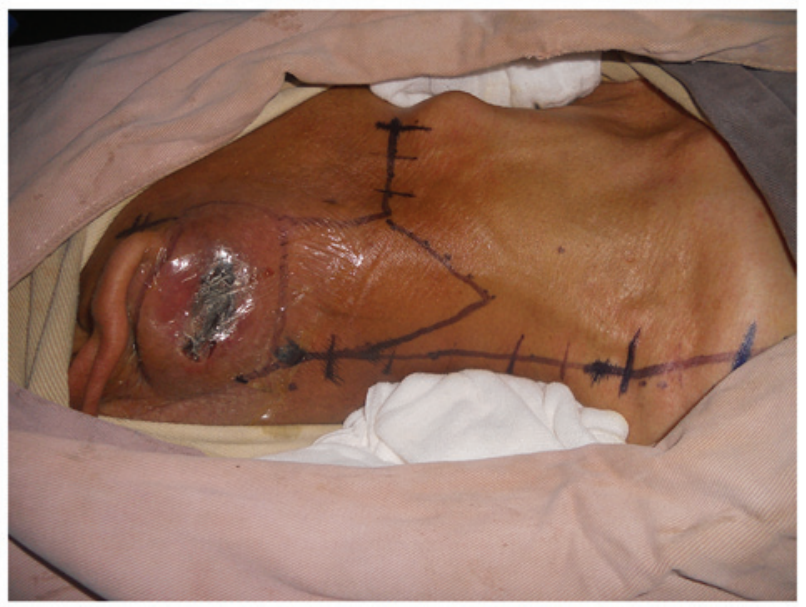

Figure 4. Skin invasion of tumor following non-standardized surgery for malignant parotid tumor.

incision opened, with ulceration, pus, local redness, swelling and pain. The patient was admitted to the Tumor Hospital of Ganzhou in February 2013. On physical examination, a tumor measuring $7.0 \times 5.0 \mathrm{~cm}$ was observed inferior to his right ear with a 5-cm-long oblique wound visible on the top of the tumor. Purulent discharge was noted in the wound, old scar formation was observed at the edge of the wound, and the tumor was firm, non-tender, ill-defined and fixed (Fig. 4). Multiple swollen lymph nodes, which ranged in diameter from 0.8 to $1 \mathrm{~cm}$ and were firm, non-tender and movable, were palpable in the right neck and supraclavicular fossa. A CT scan revealed a tumor with multiple swollen lymph nodes in the right parotid region, which indicated a diagnosis of parotid cancer. The chest radiograph revealed the presence of chronic bronchitis and emphysema, and pulmonary function tests identified severe mixed pulmonary ventilation dysfunction. Staphylococcus aureus was identified in the bacterial secretion culture, and was sensitive to cefotaxime, ceftriaxone, ampicillin and levofloxacin. On February 26th, 2013, right total parotidectomy, facial nerve buccal branch resection, right modified radical neck dissection and local flap transposition repair were performed under general anesthesia (Figs. 5-7). 


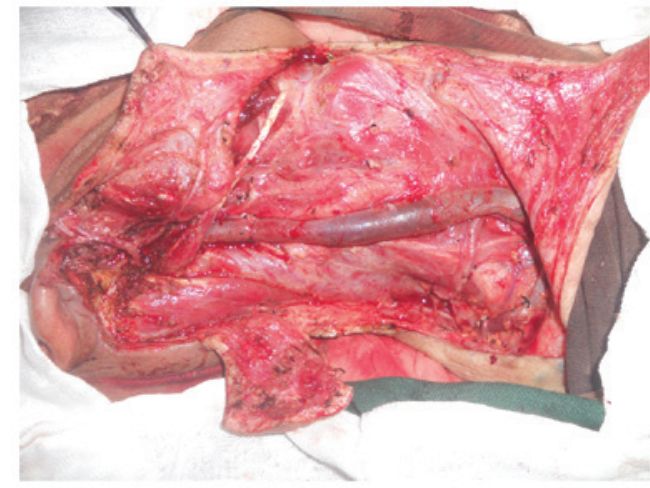

Figure 5. Total parotidectomy and resection of the buccal branch of the facial nerve with modified radical lymphadenectomy.

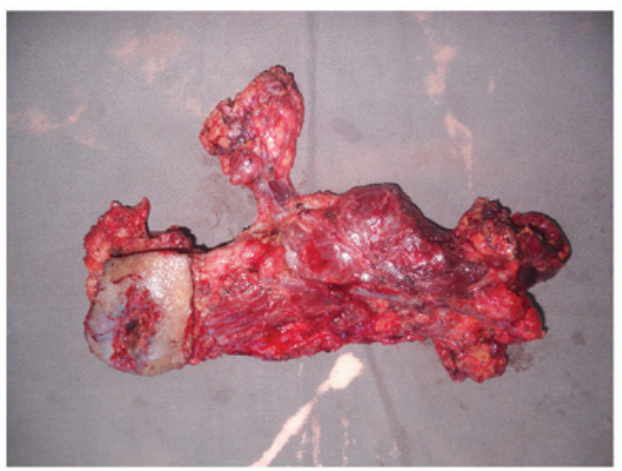

Figure 6. En bloc resected buccal branch of the facial nerve specimen excised from a patient undergoing reoperation for malignant parotid tumor.

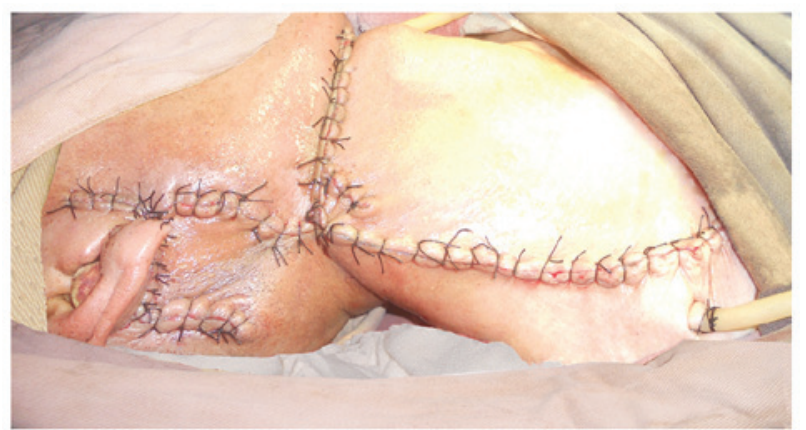

Figure 7. Appearance of the patient's neck following repair surgery of the adjacent flap.

The postoperative hispathological examination (pathological report no. 130435) revealed parotid ductal carcinoma (Fig. 8) and cervical lymph node metastases (93/95). Carcinoma cells were observed with an increased volume compared with adjacent noncarcinoma cells, with round-to-oval-shaped nuclei, coarse chromatin and distinct nucleoli, abundant cytoplasm and sieve-like or solid-nested structures were present. In addition, malignant cells also partially invaded the surrounding tissue, including muscle, nerve, neovascularization and the dermis. Partial necrosis of the trans-positioned flap was noted on March 3rd, 2013. The surgical incision was partially opened and vessels were exposed on March 5th, and pectoralis major muscle flap transposition repair was performed under general anesthesia

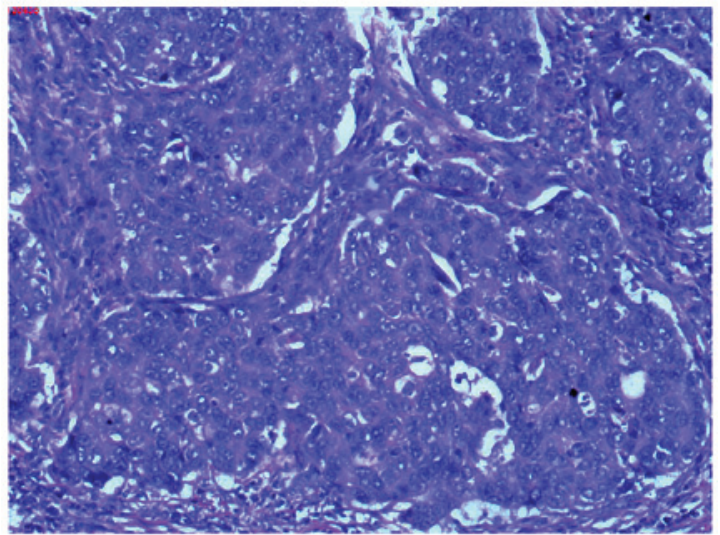

Figure 8. Hematoxylin-eosin stained parotid tumor sample (magnification, $\mathrm{x} 10$ ). The carcinoma cells exhibited an increased volume compared with the adjacent cells, with round-to-oval-shaped nuclei, coarse chromatin and distinct nucleoli. The mitotic figures were commonly observed in the cells, and abundant cytoplasm and sieve-like or solid-nested structures were present. Punctate necrosis was noted.

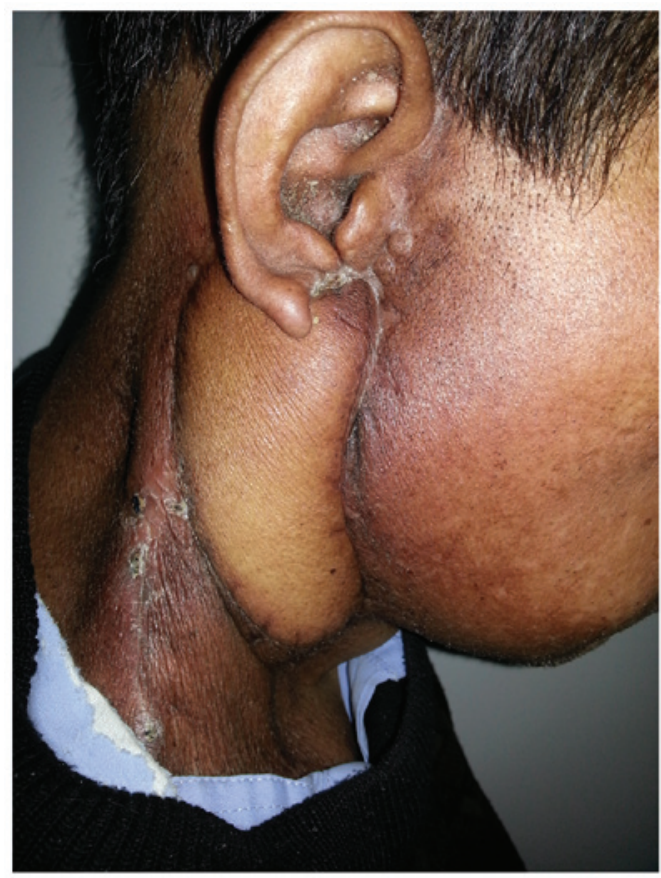

Figure 9. Partial necrosis of the adjacent flap and opened incision following pectoralis major muscle flap repair surgery and radiotherapy.

on March 6th, 2013. Conventional radiotherapy was initiated on March 25th, 2013. The radiation dose for the primary tumor site was $60 \mathrm{~Gy}$ in 30 fractions for 6 weeks and the dose for the neck lymphatic drainage region was $60 \mathrm{~Gy}$ in 30 fractions for 6 weeks. Follow-up was continued until December 2015, where it was observed that the repair flap was maintaining well (Fig. 9), and no recurrence or metastasis of the tumor were reported during this time.

\section{Discussion}

Malignant parotid tumors are the most common type of malignant salivary gland tumor, with the highest incidence 
rates (29). It is difficult to confirm the diagnosis of malignant parotid tumor prior to surgery. Malignant tumors are typically irregularly shaped, firm, ill-defined and poorly movable, and show fast growth; however, for certain painless, slow-growing tumors of the parotid gland, the possibility of malignancy cannot be easily ruled out $(30,31)$. In previous studies, the accuracy of fine-needle aspiration biopsy in the diagnosis of parotid gland tumor prior to surgery ranged from 91 to $98 \%$ (32-35). There were some misdiagnoses in pathological examination among the preoperative fine-needle aspiration biopsy specimens, intraoperative frozen sections, and paraffin-embedded sections $(31,32,36,37)$. Final diagnosis of the tumor must be confirmed by routine pathological evaluation.

It is difficult to select the most suitable procedure for the treatment of a malignant parotid tumor due to difficulties in preoperative diagnosis and the complexity of intraoperative pathological evaluation of frozen specimens (38). Consequently, partial superficial lobe parotidectomy may be initially performed for superficial lobe parotid tumors that lack accurate preoperative diagnosis; and complete superficial lobe parotidectomy may be performed in the event of suspicion of malignancy in an intraoperative frozen section. The intraoperative exploration of level II lymph nodes is required for parotid tumors suspected of being malignant based pathological examination of frozen sections. Prophylactic neck dissection is unnecessary if no positive lymph nodes are observed, while repeated frozen sectioning is required if lymph nodes are revealed during surgery to be involved. These management approaches may assist in avoiding reoperation for parotid tumors confirmed to be malignant by pathological examination of paraffin-embedded sections postoperatively (39-41).

Total parotidectomy may be performed at the time of reoperation, and the decision on whether to perform neck dissection depends on the pathological findings and extent of lymph node metastasis (42). Local adjacent flap transposition repair may be performed if there is a small area of skin invasion by the malignant parotid tumor or a low-grade tumor, and if the patient refuses free-flap or pedicle-flap repair. The submental island flap may be selected in patients without removal of sternocleidomastoid muscle in the setting of a large area of skin invasion by the tumor or a high-grade tumor (43). If neck dissection plus sternocleidomastoid excision are performed, the pectoralis major flap may be the first-line selection for skin defect repair since necrosis of the free flap, adjacent flap or submental island flap may lead to vascular exposure, increasing the risk of surgery and negatively impacting postoperative radiotherapy. The pectoralis major muscle flap comprises a large bulk of tissue that may completely repair neck tissue defects following neck dissection and cover skin defects of the parotid region (44). The pectoralis major muscle flap also has a rich blood supply, which leads to strong resistance to infection and necrosis, as well as allowing for fast healing; its application may prevent neck vascular exposure, which has been observed following necrosis of other types of flap, and will therefore not delay radiotherapy following surgery $(14,15)$. In addition, the use of the pectoralis major muscle flap allows for more natural wound appearance following surgery, and the cosmetic appearance is improved compared to those using the submental island flap (45). In the present study, 1 patient undergoing adjacent flap repair experienced flap necrosis, an opened incision site and vascular exposure, and was treated with secondary pectoralis major muscle flap transposition repair.

It is difficult to confirm the diagnosis of malignant parotid tumor prior to or during surgery. High incidence rate of residual tumor is associated with non-standardized procedures compared with standardized procedures $(46,47)$. The present study demonstrated a residual tumor rate of $63.3 \%(19 / 30)$ for patients who underwent non-standardized procedures, which was confirmed by pathological examination following reoperation. This suggests that reoperation is required in patients with malignant parotid tumor who have undergone non-standardized procedures. The Kaplan-Meier method revealed that the 5-year local control rate was $87.2 \%$, the 5-year recurrence-free survival rate was $88.1 \%$, and the 5 -year survival rate was $81.7 \%$ following reoperation. These results were similar to those of patients undergoing primary surgery (48). However, there are certain limitations to the present study. The present study was a single-center study, the sample size was small, the definitive $\mathrm{T}$ stage was missing, the follow-up durations were short in certain patients, and there may be a certain bias in the local control rate, recurrence-free survival rate and overall survival rate due to the sample size. Multi-center studies with large sample sizes are required to support the results.

With the development of head and neck functional surgery, parotid tumor surgery has evolved from the initial tumor enucleation to superficial lobe parotidectomy or total parotidectomy with facial nerve preservation, and further to the current recommended method of partial parotidectomy for benign parotid tumors $(49,50)$. In addition, with extensive application of frozen section pathological examination and the improvement of diagnostic techniques, non-standardized procedures may decrease under strict compliance with recommended surgical protocols.

\section{Acknowledgements}

The authors would like to thank the Health and Family Planning Commission of Jiangxi for supporting the present study (grant no. 20133218).

\section{References}

1. Namboodiripad PC: A review: Immunological markers for malignant salivary gland tumors. J Oral Biol Craniofac Res 4: 127-134, 2014

2. Stryjewska-Makuch G, Kolebacz B, Janik MA and Wolnik A: Increase in the incidence of parotid gland tumors in the years 2005-2014. Otolaryngol Pol 71: 29-34, 2017.

3. McHugh CH, Roberts DB, El-Naggar AK, Hanna EY, Garden AS, Kies MS, Weber RS and Kupferman ME: Prognostic factors in mucoepidermoid carcinoma of the salivary glands. Cancer 118: 3928-3936, 2012

4. Ellington CL, Goodman M, Kono SA, Grist W, Wadsworth T, Chen AY, Owonikoko T, Ramalingam S, Shin DM, Khuri FR, et al: Adenoid cystic carcinoma of the head and neck: Incidence and survival trends based on 1973-2007 Surveillance, Epidemiology, and end results data. Cancer 118: 4444-4451, 2012.

5. Deschler DG and Eisele DW: Surgery for primary malignant parotid neoplasms. Adv Otorhinolaryngol 78: 83-94, 2016.

6. Spiro RH, Huvos AG and Strong EW: Cancer of the parotid gland. A clinicopathologic study of 288 primary cases. Am J Surg 130: 452-459, 1975. 
7. Chakrabarti S, Bera M, Bhattacharya PK, Chakrabarty D, Manna AK, Pathak S and Maiti K: Study of salivary gland lesions with fine needle aspiration cytology and histopothology along with immunohistochemistry. J Indian Med Assoc 108 833-836, 2010.

8. Mohammed F, Asaria J, Payne RJ and Freeman JL: Retrospective review of 242 consecutive patients treated surgically for parotid gland tumours. J Otolaryngol Head Neck Surg 37: 340-346, 2008.

9. Kaya BV,KılıçC,Özlügedik S, Tuncel Ü and CömertE: Long-term effects of parotidectomy. Eur Arch Otorhinolaryngol 273: 4579-4583, 2016

10. Chambless LB, Kistka HM, Parker SL, Hassam-Malani L, McGirt MJ and Thompson RC: The relative value of postoperative versus preoperative karnofsky performance scale scores as a predictor of survival after surgical resection of glioblastoma multiforme. J Neurooncol 121: 359-364, 2015.

11. Nobis CP, Rohleder NH, Wolff KD, Wagenpfeil S, Scherer EQ and Kesting MR: Head and neck salivary gland carcinomas-elective neck dissection, yes or no? J Oral Maxillofac Surg 72: 205-210, 2014

12. Min R, Siyi L, Wenjun Y, Ow A, Lizheng W, Minjun D and Chenping Z: Salivary gland adenoid cystic carcinoma with cervical lymph node metastasis: A preliminary study of 62 cases. Int J Oral Maxillofac Surg 41: 952-957, 2012.

13. Revenaugh PC, Knott PD, Scharpf J and Fritz MA: Simultaneous anterolateral thigh flap and temporalis tendon transfer to optimize facial form and function after radical parotidectomy. Arch Facial Plast Surg 14: 104-109, 2012.

14. Zhang X, Liu F, Lan X, Huang J, Luo K and Li S: Resection and reconstruction of giant cervical metastatic cancer using a pectoralis major muscular flap transfer: A prospective study of 16 patients. Oncol Lett 10: 372-378, 2015.

15. Emerick KS, Herr MW, Lin DT, Santos F and Deschler DG: Supraclavicular artery island flap for reconstruction of complex parotidectomy, lateral skull base, and total auriculectomy defects. JAMA Otolaryngol Head Neck Surg 140: 861-866, 2014.

16. Bendet E, Talmi YP and Kronenberg J: Preoperative electroneurography $(\mathrm{ENoG})$ in parotid surgery: Assessment of facial nerve outcome and involvement by tumor-a preliminary study. Head Neck 20: 124-131, 1998.

17. Fujita Y, Kubota A, Furukawa M, Yagi H and Tsukuda M: Parotid gland cancer treatment with facial nerve preservation. Nihon Jibiinkoka Gakkai Kaiho 113: 115-122, 2010 (In Japanese).

18. Voss PJ, Leow AM, Schulze D, Metzger MC, Liebehenschel N and Schmelzeisen R: Navigation-guided resection with immediate functional reconstruction for high-grade malignant parotid tumour at skull base. Int J Oral Maxillofac Surg 38: 886-890, 2009.

19. Kimata Y, Sakuraba M, Hishinuma S, Ebihara S, Hayashi R and Asakage T: Free vascularized nerve grafting for immediate facial nerve reconstruction. Laryngoscope 115: 331-336, 2005.

20. Iida T, Nakagawa M, Asano T, Fukushima $C$ and Tachi K: Free vascularized lateral femoral cutaneous nerve graft with anterolateral thigh flap for reconstruction of facial nerve defects. J Reconstr Microsurg 22: 343-348, 2006.

21. House JW and Brackmann DE: Facial nerve grading system Otolaryngol Head Neck Surg 93: 146-147, 1985.

22. Sood S, McGurk M and Vaz F: Management of Salivary Gland Tumours: United Kingdom national multidisciplinary guidelines J Laryngol Otol 130: S142-S149, 2016.

23. Nagliati M, Bolner A, Vanoni V, Tomio L, Lay G, Murtas R, Deidda MA, Madeddu A, Delmastro E, Verna R, et al: Surgery and radiotherapy in the treatment of malignant parotid tumors: A retrospective multicenter study. Tumori 95: 442-448, 2009.

24. Bhide SA, Miah A, Barbachano Y, Harrington KJ, Newbold K and Nutting CM: Radical radiotherapy for treatment of malignant parotid tumours: A single centre experience 1995-2005. Br J Oral Maxillofac Surg 47: 284-289, 2009.

25. Matsuda S, Iguchi H, Tada T, Hosono M, Osawa M, Kuwae Y, Morimoto H, Okazaki E, Amano K, Miki Y, et al: Results of surgery plus postoperative radiotherapy for patients with malignant parotid tumor. Jpn J Radiol 33: 533-537, 2015.

26. Herman MP, Amdur RJ, Werning JW, Dziegielewski P, Morris CG and Mendenhall WM: Elective neck management for squamous cell carcinoma metastatic to the parotid area lymph nodes. Eur Arch Otorhinolaryngol 273: 3875-3879, 2016.

27. Jensen AD, Nikoghosyan AV, Poulakis M, Höss A, Haberer T, Jäkel O, Münter MW, Schulz-Ertner D, Huber PE and Debus J: Combined intensity-modulated radiotherapy plus raster-scanned carbon ion boost for advanced adenoid cystic carcinoma of the head and neck results in superior locoregional control and overall survival. Cancer 121: 3001-3009, 2015.
28. Qi XS, Ruan D, Lee SP, Pham A, Kupelian P, Low DA, Steinberg $M$ and Demarco J: Dependence of achievable plan quality on treatment technique and planning goal refinement: A head-and-neck intensity modulated radiation therapy application. Int J Radiat Oncol Biol Phys 91: 817-824, 2015.

29. Stodulski D, Mikaszewski B and Stankiewicz C: Are all prognostic factors in parotid gland carcinoma well recognized? Eur Arch Otorhinolaryngol 269: 1019-1025, 2012.

30. Nishikawa S, Kawata R, Higashino M, Lee K, Terada T, Kurisu Y and Tsuji M: Assessing the histological type and grade of primary parotid carcinoma by fine-needle aspiration and frozen section. Auris Nasus Larynx 42: 463-468, 2015.

31. Fakhry N, Santini L, Lagier A, Dessi P and Giovanni A: Fine needle aspiration cytology and frozen section in the diagnosis of malignant parotid tumours. Int J Oral Maxillofac Surg 43: 802-805, 2014

32. Catania A, Falvo L, D'Andrea V, Biancafarina A, De Stefano M and De Antoni E: Parotid gland tumours. Our experience and a review of the literature. Chir Ital 55: 857-864, 2003.

33. Jurczyk M, Peevey JF, Vande Haar MA and Lin X: Pitfalls of fine-needle aspiration cytology of parotid membranous basal cell adenoma-A review of pitfalls in FNA cytology of salivary gland neoplasms with basaloid cell features. Diagn Cytopathol 43: 432-437, 2015

34. Iguchi H, Wada T, Matsushita N, Oishi M, Teranishi $\mathrm{Y}$ and Yamane H: Evaluation of usefulness of fine-needle aspiration cytology in the diagnosis of tumours of the accessory parotid gland: A preliminary analysis of a case series in Japan. Acta Otolaryngol 134: 768-770, 2014.

35. Fundakowski C, Castaño J, Abouyared M, Lo K, Rivera A, Ojo R, Gomez-Fernandez C, Messinger S and Sargi Z: The role of indeterminate fine-needle biopsy in the diagnosis of parotid malignancy. Laryngoscope 124: 678-681, 2014.

36. Longuet M, Nallet E, Guedon C, Depondt J, Gehanno P and Barry B: Diagnostic value of needle biopsy and frozen section histological examination in the surgery of primary parotid tumors. Rev Laryngol Otol Rhinol (Bord) 122: 51-55, 2001 (In French).

37. O'Brien CJ: Current management of benign parotid tumors-the role of limited superficial parotidectomy. Head Neck 25: 946-952, 2003.

38. Ettl T, Schwarz-Furlan S, Gosau M and Reichert TE: Salivary gland carcinomas. Oral Maxillofac Surg 16: 267-283, 2012.

39. Swoboda $\mathrm{H}$ and Franz P: Salivary gland tumors. Clinical aspects and therapy. Radiologe 34: 232-238, 1994 (In German).

40. Obaid MA and Yusuf A: Surgical management of epithelial parotid tumours. J Coll Physicians Surg Pak 14: 394-399, 2004.

41. Shah SA, Riaz U, Zubair M and Saaiq M: Surgical presentation and outcome of parotid gland tumours. J Coll Physicians Surg Pak 23: 625-628, 2013

42. Shinomiya H, Otsuki N, Yamashita D and Nibu K: Patterns of lymph node metastasis of parotid cancer. Auris Nasus Larynx 43: 446-450, 2016

43. Sagayaraj A, Deo RP, Azeem Mohiyuddin SM and Oommen Modayil G: Island pectoralis major myocutaneous flap: An Indian perspective. Indian J Otolaryngol Head Neck Surg 64: 270-274, 2012

44. Ioannides $C$ and Fossion E: Reconstruction of extensive defects of the parotid region: Experience with the pectoralis major and free latissimus dorsi flaps. J Craniomaxillofac Surg 25: 57-62, 1997.

45. Tripathi M, Parshad S, Karwasra RK and Singh V: Pectoralis major myocutaneous flap in head and neck reconstruction: An experience in 100 consecutive cases. Natl J Maxillofac Surg 6: 37-41, 2015

46. Ezeanolue BC: Residual and recurrent parotid gland neoplasm after surgical excision. West Afr J Med 21: 5-8, 2002

47. Becelli R, Perugini M, Mastellone P and Frati R: Surgical treatment of recurrences of pleomorphic adenoma of the parotid gland. J Exp Clin Cancer Res 20: 487-489, 2001.

48. Lima RA, Tavares MR, Dias FL, Kligerman J, Nascimento MF, Barbosa MM, Cernea CR, Soares JR, Santos IC and Salviano S: Clinical prognostic factors in malignant parotid gland tumors. Otolaryngol Head Neck Surg 133: 702-708, 2005.

49. Kaur J, Goyal S, Muzumder S, Bhasker S, Mohanti BK and Rath GK: Outcome of surgery and post-operative radiotherapy for major salivary gland carcinoma: Ten year experience from a single institute. Asian Pac J Cancer Prev 15: 8259-8263, 2014.

50. Adebiyi KE and Emmanuel MM: Neoplastic salivary gland lesions: A retrospective analysis of 135 Cases from Lagos state university teaching hospital, Ikeja, Lagos, Nigeria. West Afr J Med 33: 206-210, 2014. 